\title{
Transmuted Half Normal Distribution
}

\author{
S. Balaswamy \\ Dep. of Statistics, Indira Gandhi National Tribal University, Amarkantak, India \\ *Corresponding Author: balaswamy.stats@gmail.com
}

Available online at: www.isroset.org

Accepted 19/Aug/2018, Online 30/Aug/2018

\begin{abstract}
The accumulation of large amounts of data nowadays and the necessity to characterize it leads to the birth of new statistical distributions. The current paper focuses on proposing a new distribution based on the quadratic rank transmutation map using the half normal distribution. Further, properties such as moments, Estimation of parameters, order statistics are derived for the proposed distribution along with the hazard and survival functions.
\end{abstract}

Keywords - Half Normal Distribution, Transmuted Distributions, Maximum Likelihood Estimation, Reliability Function, Quantile Function.

\section{Introduction}

Nowadays, data is obtained from various sources with multiple dimensions due to the improvement of technology, advancement of science and increasing level of ideology in research over many disciplines. In recent studies, it has been identified that many well known distributions used to model and fit the data sets do not provide enough flexibility to have an adequate fit [1]. This motivates the researchers to work on proposing new distributions in reliability and statistical theory.

One of the well known distributions is the Half Normal (HN) distribution [2] which has been used in a variety of applications. Manufacturing industries have utilized the half normal distribution to model lifetime processes under fatigue. These industries often produce goods with a long lifetime need for customers, making the cost of the resources needed to analyze the product failure times very high. To save time and money the half normal distribution is used in this reliability analysis to study the probabilistic aspects of the product failure times [3].

Therefore, half normal distribution is used to define a new transmuted distribution based on quadratic rank transmutation map [4]. This distribution will have the flexibility in fitting lifetime processes under fatigue and will even have the adequate fit for the skewed data.

A random variable ' $\mathrm{X}$ ' is said to have transmuted distribution if its cumulative distribution function (cdf) and probability density function (pdf) are given by (1) and (2).

$G(x)=(1+\lambda) F(x)-\lambda F(x)^{2}$ $g(x)=f(x)[(1+\lambda)-2 \lambda F(x)]$

where $\mathrm{f}(\mathrm{x})$ and $\mathrm{F}(\mathrm{x})$ are the probability density function (pdf) $\&$ cumulative distribution function (cdf) of the subject distribution and $\lambda$ is the transmuted parameter $(-1 \leq \lambda \leq 1)$.

The focus of this paper is to derive a new Transmuted Half Normal (THN) distribution using quadratic rank transmutation map.

\section{The Transmuted Half Normal Distribution}

Bland and Altman [2] used the half normal distribution to study the relationship between measurement error and magnitude. Bland [5] extended the work of Bland and Altman [2] by using the distribution to estimate the standard deviation as a function so that measurement error could be controlled. The cumulative distribution function and probability density function of the half normal distribution is given in (3) and (4).

$F(x)=\operatorname{erf}\left(\frac{x}{\sigma \sqrt{2}}\right)$

$f(x)=\frac{1}{\sigma} \sqrt{\frac{2}{\pi}} \exp \left(\frac{-x^{2}}{2 \sigma^{2}}\right)$

where $\sigma$ is the scale parameter and $\operatorname{erf}(\cdot)$ is the error function.

A random variable $X$ is said to have the transmuted half normal distribution (THND) with parameter $\sigma$ and $\lambda$ and -1 $\leq \lambda \leq 1$ and its cumulative distribution function is defined using equations (1) and (3) as follows,

$G(x)=\operatorname{erf}\left(\frac{x}{\sigma \sqrt{2}}\right)\left[1+\lambda\left(1-\operatorname{erf}\left(\frac{x}{\sigma \sqrt{2}}\right)\right)\right]$

The probability density function of the transmuted half normal distribution is obtained by substituting (4) in (2),

$g(x)=\frac{1}{\sigma} \sqrt{\frac{2}{\pi}} \exp \left(\frac{-x^{2}}{2 \sigma^{2}}\right)\left[1+\lambda\left(1-2 \operatorname{erf}\left(\frac{x}{\sigma \sqrt{2}}\right)\right)\right]$ (6) 
where $\sigma$ is the scale parameter and $\lambda$ is the transmutation parameter. The half normal distribution is a special case for $\lambda=0$.

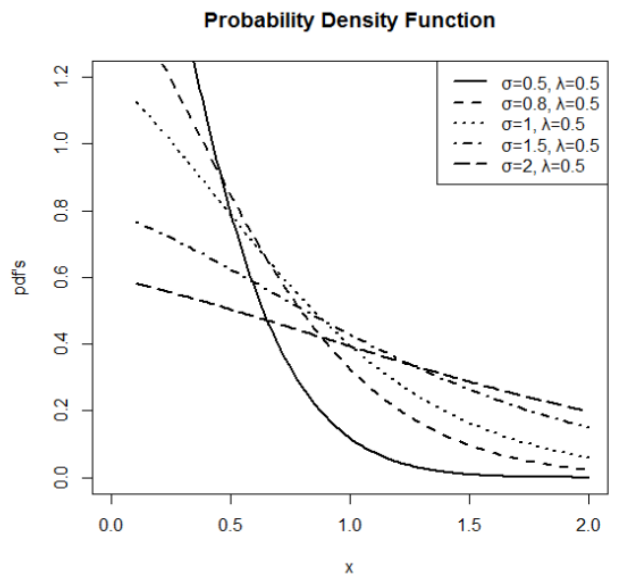

Probability Density Function

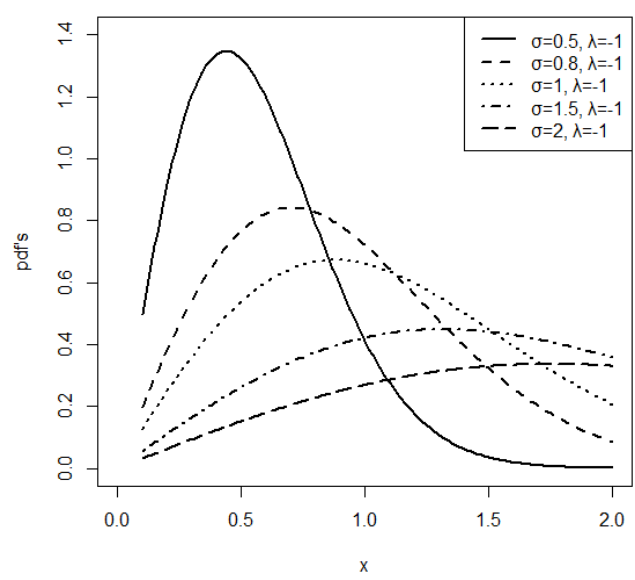

The probability density and cumulative distribution functions of THND will exhibit different behavior depending on the values of the parameters when chosen to be positive (Figures $1 \& 2$ ).
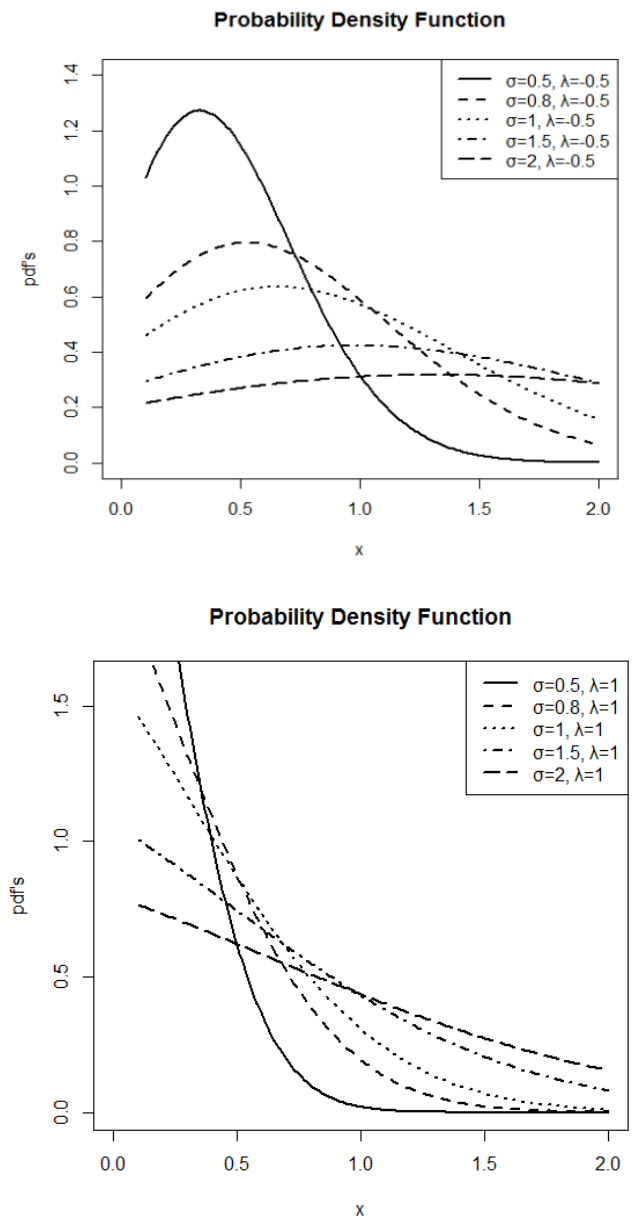

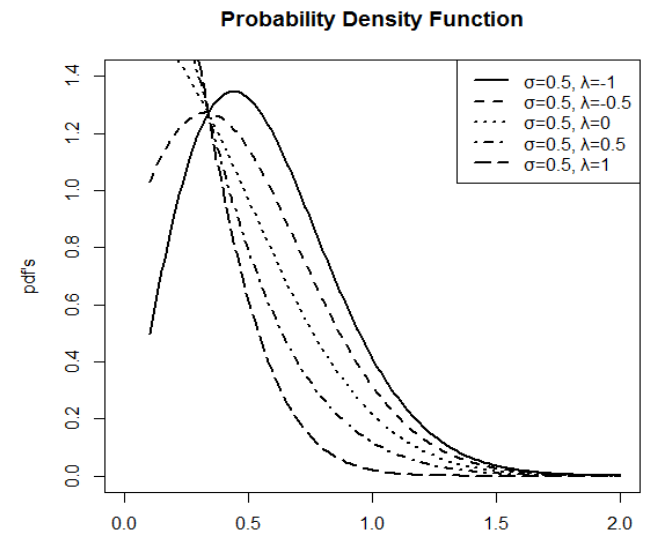

Figure 1: Probability Density Function of the Transmuted Half Normal Distribution 

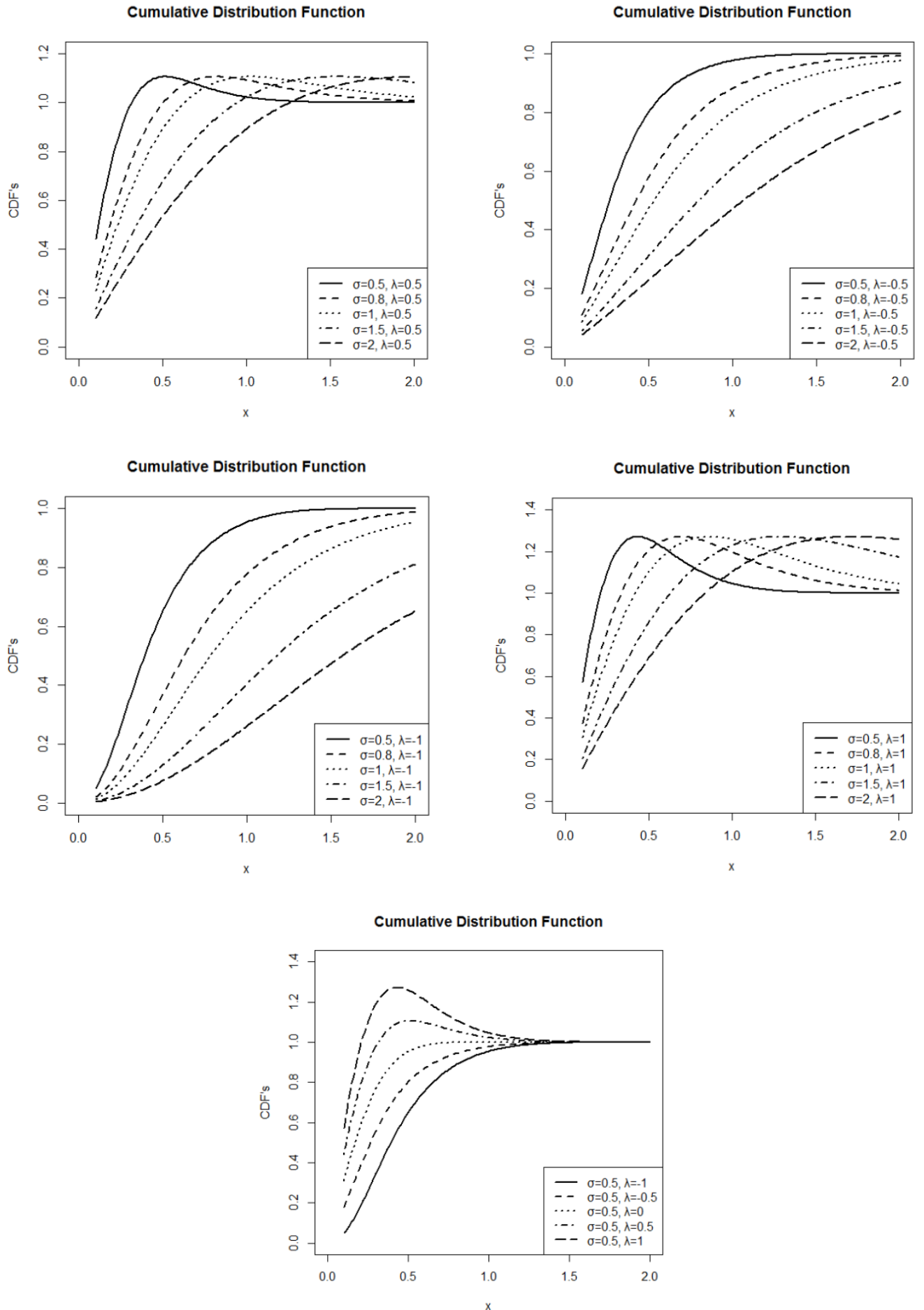

Figure 2: Cumulative Distribution Function of the Transmuted Half Normal Distribution

\section{Reliability Analysis}

In this section, the survival and hazard functions for THND are presented.

\subsection{Survival Function}

The transmuted half normal distribution can be used to characterize the failure time of a given system. The reliability (survival) function $\mathrm{S}(\mathrm{x})$, which is the probability of an item not failing prior to sometime ' $t$ ', is defined as

$$
S(x)=1-G(x)
$$


Therefore, the survival function for the transmuted half normal distribution is defined in equation (7) as,

$$
S(x)=1-\operatorname{erf}\left(\frac{x}{\sigma \sqrt{2}}\right)\left[1+\lambda\left(1-\operatorname{erf}\left(\frac{x}{\sigma \sqrt{2}}\right)\right)\right]
$$

on further simplification using the relationship between standard normal distribution and error function, the expression for survival function is

$$
S(x)=4 \lambda\left[\Phi\left(\frac{x}{\sigma}\right)\right]^{2}-6 \lambda \Phi\left(\frac{x}{\sigma}\right)-2 \Phi\left(\frac{x}{\sigma}\right)+2 \lambda+2
$$

\subsection{Hazard Function}

The hazard rate function of the THND is defined as follows,

$$
\begin{gathered}
h(x)=\frac{g(x)}{1-G(x)} \\
h(x)=\frac{\frac{1}{\sigma} \sqrt{\frac{2}{\pi}} \exp \left(\frac{-x^{2}}{2 \sigma^{2}}\right)\left[1+\lambda\left(1-2 \operatorname{erf}\left(\frac{x}{\sigma \sqrt{2}}\right)\right)\right]}{1-\operatorname{erf}\left(\frac{x}{\sigma \sqrt{2}}\right)\left[1+\lambda\left(1-\operatorname{erf}\left(\frac{x}{\sigma \sqrt{2}}\right)\right)\right]}
\end{gathered}
$$

on further simplification, the expression for hazard function as follows,

$h(x)=\left[\frac{\exp \left(\frac{-x^{2}}{2 \sigma^{2}}\right)}{\sigma \sqrt{2 \pi}}\right]\left[\frac{3 \lambda-4 \lambda \Phi\left(\frac{x}{\sigma}\right)+1}{2 \lambda\left[\Phi\left(\frac{x}{\sigma}\right)\right]^{2}-3 \lambda \Phi\left(\frac{x}{\sigma}\right)-\Phi\left(\frac{x}{\sigma}\right)+\lambda+1}\right]$

Here, the different cases of the hazard function are given below:

Case 1: when $\lambda=1$, the hazard function in equation (8) reduces to

$$
h(x)=\left[\frac{\exp \left(\frac{-x^{2}}{2 \sigma^{2}}\right)}{\sigma \sqrt{2 \pi}}\right]\left[\frac{2}{1-\Phi\left(\frac{x}{\sigma}\right)}\right]
$$

Case 2: when $\lambda=0$, the hazard function in equation (8) reduces to

$$
h(x)=\left[\frac{\exp \left(\frac{-x^{2}}{2 \sigma^{2}}\right)}{\sigma \sqrt{2 \pi}}\right]\left[\frac{1}{2}\left(1-\operatorname{erf}\left(\frac{x}{\sigma \sqrt{2}}\right)\right)\right]
$$

Case 3: when $\lambda=1$ and $\sigma=1$, the hazard function in equation (8) reduces to

$$
h(x)=\left[\frac{\exp \left(\frac{-x^{2}}{2}\right)}{\sqrt{2 \pi}}\right]\left[\frac{2}{1-\Phi(x)}\right]
$$

Case 4: when $\mathrm{x}=0$, the hazard function in equation (8) reduces to

$$
h(x)=\left[\frac{2(1+\lambda)}{\sigma \sqrt{2 \pi}}\right]
$$

Case 5: when $x=\infty$, then $h(\infty)=0$.

When modeling monotone hazard rates, the HN and THN distributions might be the initial choices because of their negatively and positively skewed density shapes. However, they do not provide a reasonable parametric fit for modeling phenomenon with non-monotone failure rates such as the bathtub shaped curve (Figure 3). Such bathtub hazard curves have nearly flat middle portions and the corresponding densities have a positive anti mode (Figure 1). Therefore, the hazard function can have many of the different shapes as shown in Figure 3.

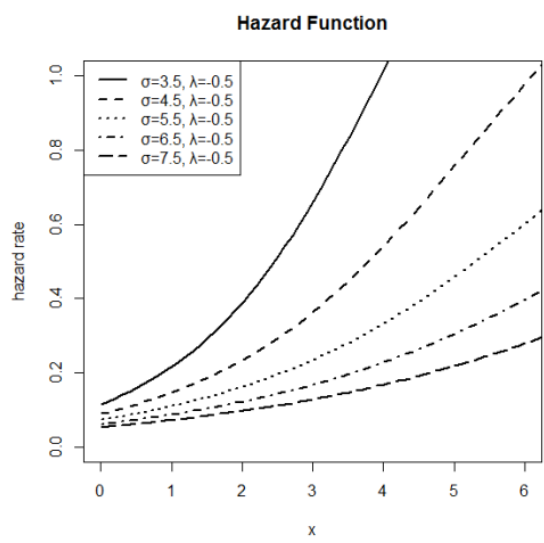



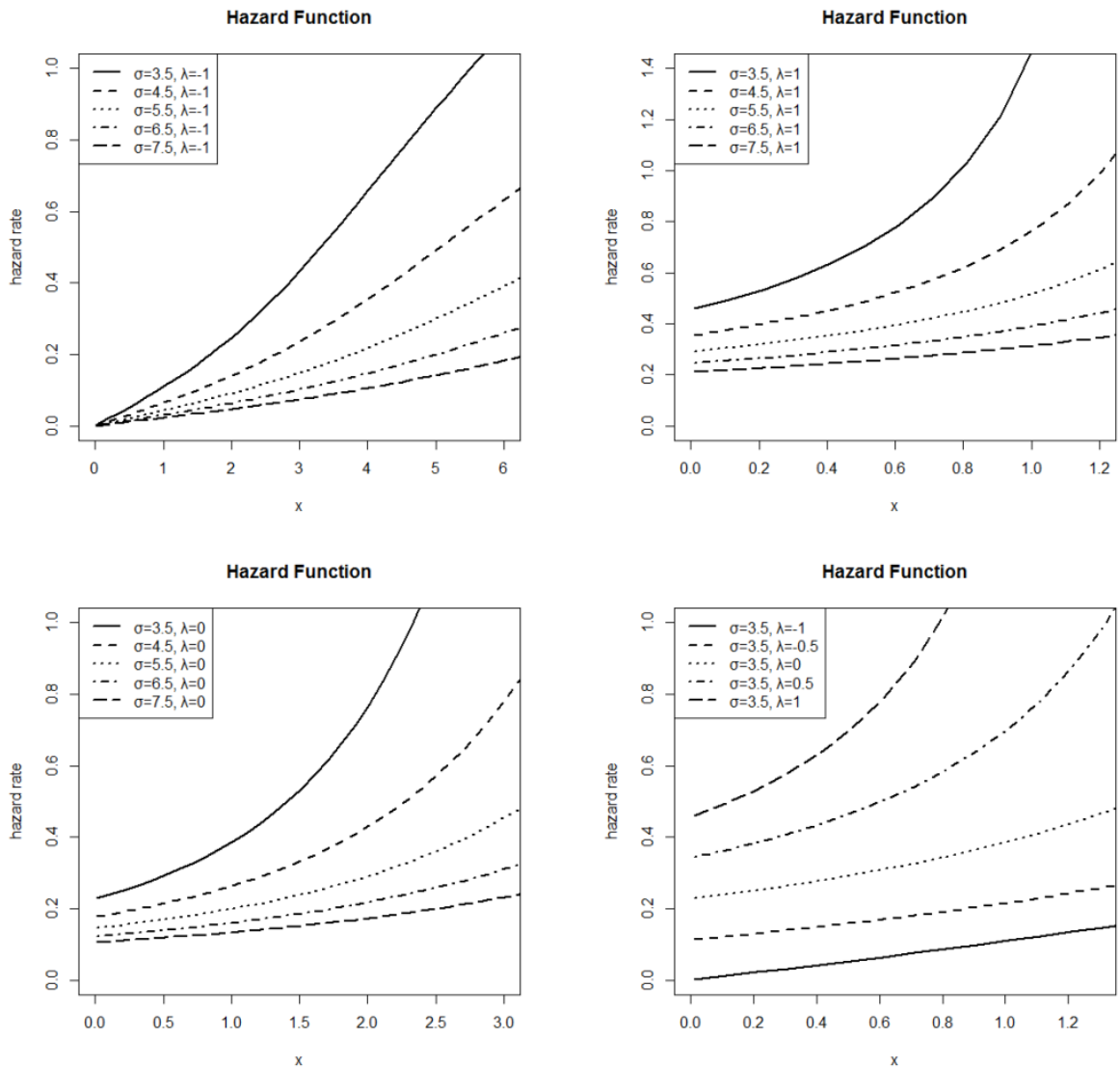

Figure 3: Hazard Function of the Transmuted Half Normal Distribution Consider $\int_{0}^{\infty} x^{k} \exp \left(\frac{-x^{2}}{2 \sigma^{2}}\right) d x$.

\section{Statistical Properties}

In this section, statistical properties of THND using moments are discussed.

\subsection{Moments}

It is necessary to provide the mean and variance through the method of moments when a new distribution is proposed. Therefore, the explicit expressions for the $\mathrm{k}^{\text {th }}$ order moments are as follows,

$$
\begin{gathered}
E\left(x^{k}\right)=\int_{0}^{\infty} x^{k} g(x) d x \\
E\left(x^{k}\right)=\int_{0}^{\infty} x^{k} \frac{1}{\sigma} \sqrt{\frac{2}{\pi}} \exp \left(\frac{-x^{2}}{2 \sigma^{2}}\right)\left[1+\lambda\left(1-2 \operatorname{erf}\left(\frac{x}{\sigma \sqrt{2}}\right)\right)\right] d x \\
E\left(x^{k}\right)=\frac{1}{\sigma} \sqrt{\frac{2}{\pi}} \int_{0}^{\infty} x^{k} \exp \left(\frac{-x^{2}}{2 \sigma^{2}}\right) d x+\frac{\lambda}{\sigma} \sqrt{\frac{2}{\pi}} \int_{0}^{\infty} x^{k} \exp \left(\frac{-x^{2}}{2 \sigma^{2}}\right) d x- \\
\frac{2 \lambda}{\sigma} \sqrt{\frac{2}{\pi}} \int_{0}^{\infty} x^{k} \exp \left(\frac{-x^{2}}{2 \sigma^{2}}\right) \operatorname{erf}\left(\frac{x}{\sigma \sqrt{2}}\right) d x
\end{gathered}
$$

Let us make the transformation by assuming $z=\frac{x}{\sigma \sqrt{2}}$ then $d x=\sigma \sqrt{2} d z$. Therefore, the following expression is to be solved,

$$
\begin{aligned}
\int_{0}^{\infty} x^{k} \exp \left(\frac{-x^{2}}{2 \sigma^{2}}\right) d x & =\left(2 \sigma^{2}\right)^{\frac{k}{2}} \sigma \sqrt{2} \int_{0}^{\infty} z^{k} \exp \left(-z^{2}\right) d z \\
= & \sigma^{k} 2^{\frac{k}{2}} \frac{\sigma \sqrt{2}}{2} \Gamma\left(\frac{k+1}{2}\right) \\
\int_{0}^{\infty} x^{k} \exp \left(\frac{-x^{2}}{2 \sigma^{2}}\right) d x & =\sigma^{(k+1)} 2^{\frac{(k-1)}{2}} \frac{\sigma \sqrt{2}}{2} \Gamma\left(\frac{k+1}{2}\right)
\end{aligned}
$$

Now consider, $\int_{0}^{\infty} x^{k} \exp \left(\frac{-x^{2}}{2 \sigma^{2}}\right) \operatorname{erf}\left(\frac{x}{\sigma \sqrt{2}}\right) d x$.

Again using the same transformation $z=\frac{x}{\sigma \sqrt{2}}$ which implies that $d x=\sigma \sqrt{2} d z$. Therefore, one can solve the following expression, 


$$
\begin{aligned}
& \int_{0}^{\infty} x^{k} \exp \left(\frac{-x^{2}}{2 \sigma^{2}}\right) \operatorname{erf}\left(\frac{x}{\sigma \sqrt{2}}\right) d x \\
& =(\sigma \sqrt{2})^{k} \sigma \sqrt{2} \int_{0}^{\infty} z^{k} \exp \left(-z^{2}\right) \operatorname{erf}(z) d z \\
& =\sigma \sqrt{\frac{2}{\pi}}\left(2 \sigma^{2}\right)^{\frac{k}{2}} \frac{2}{1} F\left(\frac{1}{2}, \frac{k+2}{2}, \frac{3}{2} ;-1\right) \Gamma\left(\frac{k}{2}+1\right)
\end{aligned}
$$

where $\Gamma(\cdot)$ is the gamma function and ${ }_{1}^{2} F(\cdot)$ is the hyper geometric function.

Therefore, the expression in equation (9) can be rewritten using (10) and (11) as follows,

$$
\begin{aligned}
& E\left(x^{k}\right) \\
& =\frac{1}{\sigma} \sqrt{\frac{2}{\pi}} \sigma^{(k+1)} 2^{\frac{(k-1)}{2}} \frac{\sigma \sqrt{2}}{2} \Gamma\left(\frac{k+1}{2}\right) \\
& +\frac{\lambda}{\sigma} \sqrt{\frac{2}{\pi}} \sigma^{(k+1)} 2^{\frac{(k-1)}{2}} \frac{\sigma \sqrt{2}}{2} \Gamma\left(\frac{k+1}{2}\right) \\
& -\frac{2 \lambda}{\sigma} \sqrt{\frac{2}{\pi}} \sigma \sqrt{\frac{2}{\pi}}\left(2 \sigma^{2}\right)^{\frac{k}{2}}{ }_{1}^{2} F\left(\frac{1}{2}, \frac{k+2}{2}, \frac{3}{2} ;-1\right) \Gamma\left(\frac{k}{2}+1\right)
\end{aligned}
$$

on further simplification,

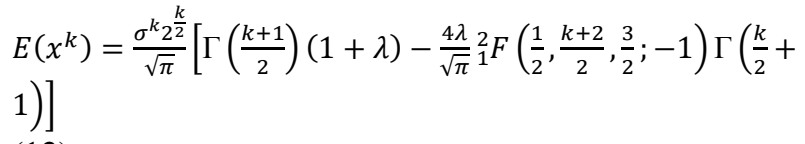

When $\lambda=0$ in equation (12), the $\mathrm{k}^{\text {th }}$ order moment for the half normal distribution is as,

$$
E\left(x^{k}\right)=\frac{\sigma^{k} 2^{\frac{k}{2}}}{\sqrt{\pi}} \Gamma\left(\frac{k+1}{2}\right)
$$

when $\mathrm{k}=1$, then the mean of the transmuted half normal distribution is given by,

$E(x)=\sigma \sqrt{\frac{2}{\pi}}\left[1-\lambda\left(\frac{\pi}{2}-1\right)\right]$

If $\lambda=0$, then the mean of the half normal distribution [5] is given by,

$$
E(x)=\sigma \sqrt{\frac{2}{\pi}}
$$

when $\mathrm{k}=2$, then the second order moment of the transmuted half normal distribution is given by,

$$
E\left(x^{2}\right)=\frac{\sigma^{2} 2}{\sqrt{\pi}}\left[\frac{\sqrt{\pi}}{2}-0.7096 \lambda\right]
$$

Further, the variance of the transmuted half normal distribution is

$$
\begin{aligned}
& \operatorname{Var}(x)= \\
& \frac{\sigma^{2} 2}{\sqrt{\pi}}\left[\frac{\sqrt{\pi}}{2}-0.7096 \lambda\right]- \\
& \left.\left.\lambda\left(\frac{\pi}{2}-1\right)\right]\right]^{2}
\end{aligned}
$$$$
\left[\sigma \sqrt{\frac{2}{\pi}}[1-\right.
$$

If $\lambda=0$, then the variance of the half normal distribution [5] is given by,

$$
\operatorname{Var}(x)=\sigma^{2}\left(1-\frac{2}{\pi}\right)
$$

\section{Quantile Function}

The quantile function returns the threshold value ' $\mathrm{x}$ ' for a given value $\mathrm{q}$ such that $\mathrm{F}(\mathrm{x})=\mathrm{q}(0 \leq \mathrm{q} \leq 1)$. The quantile function helps in explaining the fit for a given data set to a theoretical distribution. Here, the quantile function for the corresponding transmuted half normal distribution (5) is given by,

$x_{q}=\sigma \sqrt{2} \operatorname{erf}^{-1}\left(\frac{-(\lambda+1) \pm \sqrt{\lambda^{2}+2 \lambda(1-2 q)+1}}{2 \lambda}\right)$

in particular, the median of the THN distribution is,

$x_{q}=\sigma \sqrt{2} \operatorname{erf}^{-1}\left(\frac{-(\lambda+1) \pm \sqrt{\lambda^{2}+1}}{2 \lambda}\right)$

\section{Maximum Likelihood Estimation}

Let $x_{1}, x_{2}, \cdots, x_{n}$ be a sample of size ' $\mathrm{n}$ ' from transmuted half normal distribution. Then the likelihood function ' $L$ ' is given by,

$$
L=\left(\sigma \sqrt{\frac{2}{\pi}}\right)^{n} \exp \left(-\sum_{i=1}^{n} \frac{x_{i}^{2}}{2 \sigma^{2}}\right) \prod_{i=1}^{n}\left[1+\lambda\left[1-2 \operatorname{erf}\left(\frac{x}{\sigma \sqrt{2}}\right)\right]\right]
$$

Hence, the log-likelihood function is given by,

$$
\begin{aligned}
\log L=n \log \left(\sigma \sqrt{\frac{2}{\pi}}\right)-\left(\sum_{i=1}^{n} \frac{x_{i}^{2}}{2 \sigma^{2}}\right) \\
+\sum_{i=1}^{n} \log \left[1+\lambda\left[1-2 \operatorname{erf}\left(\frac{x}{\sigma \sqrt{2}}\right)\right]\right]
\end{aligned}
$$

on further simplification, the log-likelihood function is as follows 


$$
\begin{gathered}
\log L=n \log (\sqrt{2})-n \log (\sigma \sqrt{\pi})- \\
\frac{1}{2 \sigma^{2}}\left(\sum_{i=1}^{n} x_{i}^{2}\right)+\sum_{i=1}^{n} \log \left[1+3 \lambda-4 \lambda \Phi\left(\frac{x_{i}}{\sigma}\right)\right]
\end{gathered}
$$

The partial differentiation with respect to the parameters of log-likelihood function are given as follows,

$$
\begin{aligned}
& \frac{\partial \log L}{\partial \sigma}=\frac{-n}{\sigma}+\frac{1}{\sigma^{3}} \sum_{i=1}^{n} x_{i}^{2}+\frac{4 \lambda}{\sigma^{2}} \sum_{i=1}^{n} \frac{x_{i} \phi\left(\frac{x_{i}}{\sigma}\right)}{\left[1+3 \lambda-4 \lambda \Phi\left(\frac{x_{i}}{\sigma}\right)\right]} \\
& \frac{\partial \log L}{\partial \lambda}=\sum_{i=1}^{n} \frac{3-4 \Phi\left(\frac{x_{i}}{\sigma}\right)}{\left[1+3 \lambda-4 \lambda \Phi\left(\frac{x_{i}}{\sigma}\right)\right]}
\end{aligned}
$$

Therefore, the MLE's of $\sigma$ and $\lambda$ which maximizes the equation (19) must satisfy the following normal equations,

$$
\begin{gathered}
\frac{-n}{\sigma}+\frac{1}{\sigma^{3}} \sum_{i=1}^{n} x_{i}^{2}+\frac{4 \lambda}{\sigma^{2}} \sum_{i=1}^{n} \frac{x_{i} \phi\left(\frac{x_{i}}{\sigma}\right)}{\left[1+3 \lambda-4 \lambda \Phi\left(\frac{x_{i}}{\sigma}\right)\right]}=0 \\
\sum_{i=1}^{n} \frac{3-4 \Phi\left(\frac{x_{i}}{\sigma}\right)}{\left[1+3 \lambda-4 \lambda \Phi\left(\frac{x_{i}}{\sigma}\right)\right]}=0
\end{gathered}
$$

Here, the maximum likelihood estimates of $\sigma$ and $\lambda$ are obtained by solving the above non-linear system of equations (22). Hence, the Newton - Raphson's algorithm is useful to obtain the maximum likelihood estimates of $\sigma$ and $\lambda$ by directly maximizing the log-likelihood function given in equation (19).

In order to compute the standard errors and asymptotic confidence intervals, the usual large sample approximation in which the maximum likelihood estimators of $\theta=$ $(\hat{\sigma}, \hat{\lambda})$ can be approximated to $N\left(0, I^{-1}(\hat{\theta})\right)$.

Where $I(\theta)=E\left[\frac{\partial^{2} \log L}{\partial \theta \partial \theta^{\prime}}\right]$. This information matrix I $(\theta)$ can be approximated by the observed information matrix as follows,

$$
\begin{gathered}
I(\hat{\theta})=E\left[\frac{\partial^{2} \log L}{\partial \theta \partial \theta^{\prime}}\right]_{\theta=\theta^{\prime}}=\left(\begin{array}{ll}
A_{11} & A_{12} \\
A_{21} & A_{22}
\end{array}\right)_{\theta=\theta^{\prime}}^{-1} \\
=\left(\begin{array}{ll}
I_{11} & I_{12} \\
I_{21} & I_{22}
\end{array}\right)_{\theta=\theta^{\prime}}
\end{gathered}
$$

where $A_{11}=-\frac{\partial^{2} \log L}{\partial \sigma^{2}} ; A_{12}=A_{21}=-\frac{\partial^{2} \log L}{\partial \sigma \partial \lambda}$

and $A_{22}=-\frac{\partial^{2} \log L}{\partial \lambda^{2}}$.

By using the approximation $\sqrt{n}(\hat{\theta}-\theta) \sim N\left(0, I^{-1}(\hat{\theta})\right)$, the confidence intervals for the parameters of $\theta$ can be obtained as follows,

$$
\hat{\sigma} \pm Z \alpha / 2 \sqrt{\hat{I}_{11}} \text { and } \hat{\lambda} \pm Z \alpha / 2 \sqrt{\hat{I}_{22}}
$$

where $Z_{\alpha / 2}$ is the $\alpha / 2$ percentile of standard normal distribution.

\section{Random Number Generation}

The random numbers can be generated for the transmuted half normal distribution using the method of inversion as follows,

$$
\operatorname{erf}\left(\frac{x}{\sigma \sqrt{2}}\right)\left[1+\lambda\left(1-\operatorname{erf}\left(\frac{x}{\sigma \sqrt{2}}\right)\right)\right]=u
$$

on further simplification, the second order equation is as follows

$$
\lambda\left[\operatorname{erf}\left(\frac{x}{\sigma \sqrt{2}}\right)\right]^{2}-(\lambda+1) \operatorname{erf}\left(\frac{x}{\sigma \sqrt{2}}\right)+u=0
$$

The roots of the above equation (23)are as follows,

$$
x=\sigma \sqrt{2} \operatorname{erf}^{-1}\left(\frac{-(\lambda+1) \pm \sqrt{\lambda^{2}+2 \lambda(1-2 u)+1}}{2 \lambda}\right)
$$

\section{Order Statistics}

If $X_{(1)} \leq X_{(2)} \leq \cdots \leq X$ denote the order statistics of a random sample $X_{1}, X_{2}, \cdots, X_{n}$ from a continuous population with the $\operatorname{cdf} G_{X}(x)$ and pdf $g_{X}(x)$ then the pdf of $X_{(j)}$ is given by

$$
g X_{(j)}(x)=\frac{n !}{(j-1) !(n-j) !} g_{X}(x)\left[G_{X}(x)\right]^{j-1}\left[1-G_{X}(x)\right]^{n-j}
$$

The pdf of the $\mathrm{j}^{\text {th }}$ order statistic from THN distribution is,

$$
\begin{gathered}
g X_{(j)}(x)=\frac{n !}{(j-1) !(n-j) !} \frac{1}{\sigma} \sqrt{\frac{2}{\pi}} \exp \left(\frac{-x^{2}}{2 \sigma^{2}}\right)\left[1+\lambda\left(1-2 \operatorname{erf}\left(\frac{x}{\sigma \sqrt{2}}\right)\right)\right] \\
{\left[\operatorname{erf}\left(\frac{x}{\sigma \sqrt{2}}\right)\left[1+\lambda\left(1-\operatorname{erf}\left(\frac{x}{\sigma \sqrt{2}}\right)\right)\right]\right]^{j-1}[1} \\
\left.-\left[\operatorname{erf}\left(\frac{x}{\sigma \sqrt{2}}\right)\left[1+\lambda\left(1-\operatorname{erf}\left(\frac{x}{\sigma \sqrt{2}}\right)\right)\right]\right]\right]^{n-j}
\end{gathered}
$$

The pdf of the largest order statistic is given by,

$$
\begin{gathered}
g X_{(j)}(x)=\frac{n}{\sigma} \sqrt{\frac{2}{\pi}} \exp \left(\frac{-x^{2}}{2 \sigma^{2}}\right)\left[1+\lambda\left(1-2 \operatorname{erf}\left(\frac{x}{\sigma \sqrt{2}}\right)\right)\right] \\
{\left[\operatorname{erf}\left(\frac{x}{\sigma \sqrt{2}}\right)\left[1+\lambda\left(1-\operatorname{erf}\left(\frac{x}{\sigma \sqrt{2}}\right)\right)\right]\right]^{n-1}}
\end{gathered}
$$

The pdf of the smallest order statistic is given by, 


$$
\begin{gathered}
g X_{(j)}(x)=\frac{n}{\sigma} \sqrt{\frac{2}{\pi}} \exp \left(\frac{-x^{2}}{2 \sigma^{2}}\right)\left[1+\lambda\left(1-2 \operatorname{erf}\left(\frac{x}{\sigma \sqrt{2}}\right)\right)\right] \\
{\left[1-\left[\operatorname{erf}\left(\frac{x}{\sigma \sqrt{2}}\right)\left[1+\lambda\left(1-\operatorname{erf}\left(\frac{x}{\sigma \sqrt{2}}\right)\right)\right]\right]\right]^{n-1}}
\end{gathered}
$$

\section{Conclusion}

In this paper, a new distribution namely, the transmuted half normal distribution has been introduced. The subject distribution is derived using quadratic rank transformation map. Statistical properties such as moments and quantile functions are presented. Further, estimation of parameters of THND are obtained using maximum likelihood estimation procedure. The behavior of THND is studied using the hazard and survival functions. Further, it is seen that the transmuted half normal distribution can be used to assess the data with adequate fit when data is of lifetime processes under fatigue.

\section{References}

[1]. Ayman Alzaatreh and Kristen Knight. On The Gamma-Half Normal Distribution and Its Applications. Journal of Modern Applied Statistical Methods, 12 (2013), no. 1, 103-119.

[2]. Bland, J. M. and Altman, D. G. Measuring agreement in method comparison studies. Statistical Methods in Medical Research, 8 (1999), 135-160.

[3]. Castro, L., Gomez, H., and Valenzuela, M. (2012). Epsilon half normal model: properties and inference. Computational Statistics and Data Analysis, 56 (2012), no. 12, 4338-4347.

[4]. Shaw W.T. and Buckley I.R.C. The alchemy of probability distributions: beyond gram-charlier expansions, and a skewkurtotic-normal distribution from a rank transmutation map. arXiv preprint, page arXiv:0901.0434, (2007).

[5]. Bland, J. M. The half-normal distribution method for measurement error: two case studies. Unpublished lecture available on http://wwwusers.york.ac.uk/ mb55/talks/halfnor.pdf. (2005).

\section{AUTHORS PROFILE}

Dr. S. Balaswamy is currently working as Assistant Professor in the Department of Statistics, Indira Gandhi National Tribal University, Amarkantak. His areas of research interest are Biostatistics, Classification and Statistical Computing. He has published 16 research papers in reputed National and International peer reviewed journals. He has presented more than 10 research papers in National and International conferences/Seminars and also participated in more than 10 National and International workshops. He is a recipient of Prof. B.K. Kale Award for best paper Presentation in an International Conference. He is a life member of Indian Society for Probability and Statistics. 